\title{
Spectroscopic properties of monolithic sol-gel glasses doped with lanthanide bipyridyl complexes
}

\author{
Kris Driesen, Christiane Görller-Walrand, Koen Binnemans* \\ Department of Chemistry, Katholieke Universiteit Leuven, Celestijnenlaan 200F, B-3001 Leuven, Belgium
}

\begin{abstract}
Lanthanide complexes [ $\left.\mathrm{Ln}(\mathrm{bpy})_{2}\right] \mathrm{Cl}_{3}$ (bpy $=$ bipyridyl, 2,2'-bipyridine; $\mathrm{Ln}=\mathrm{Nd}, \mathrm{Ho}$, Er) were incorporated via a sol-gel process into a silicate glass. Optical absorption spectra of the monolithic glass samples have been recorded. The data were interpreted using the Judd-Ofelt theory. It was found that heat treatment of the glass samples results in a marked increase of the intensity of the hypersensitive transitions, whereas the intensity of the other $\mathrm{f}-\mathrm{f}$ transitions decreases slightly. These differences in spectral behaviour are mainly caused by the removal of water molecules out of the coordination sphere of the lanthanide ions and by the densification of the glass matrix. (C) 2001 Elsevier Science B.V. All rights reserved.
\end{abstract}

Keywords: Rare earths; Lanthanides; Sol-gel glasses; Spectroscopy; f-f transitions

\section{Introduction}

Via the sol-gel process, it is possible to synthesise at room temperature a porous glassy network. Due to the porosity and the low processing temperatures of sol-gel glasses, organic molecules or coordination compounds can be trapped into the sol-gel matrix [1,2]. Earlier studies of lanthanide complexes doped into sol-gel glasses were mainly devoted to the luminescence properties of europium(III) and terbium(III) complexes [3-15]. Although hundreds of sets of intensity parameters have been published for lanthanide ions in inorganic glasses [16], only very few intensity parameters are available for lanthanide complexes in sol-gel glasses or in hybrid organic-inorganic composite materials (e.g. Refs. [17,18]).

In this paper, we describe the spectroscopic properties of $\mathrm{Ln}$ (III) bipyridyl complexes [ $\mathrm{Ln}(\mathrm{III})=\mathrm{Nd}$ (III), Ho(III) and $\mathrm{Er}(\mathrm{III})]$ doped in an $\mathrm{SiO}_{2}$ sol-gel glass. The intensities of the transitions in the absorption spectra are parameterised in terms of the Judd-Ofelt intensity parameters $[19,20]$. The influence of heat treatment on the intensities is discussed.

\footnotetext{
* Corresponding author. Tel.: +32-16-32-74-46; fax: +32-16-32-79-92

E-mail address: Koen.Binnemans@ chem.kuleuven.ac.be (K. Binnemans).
}

\section{Experimental details}

The lanthanide(III) bipyridyl complexes, $\left[\mathrm{Ln}(\mathrm{bpy})_{2}\right] \mathrm{Cl}_{3}$ - $2 \mathrm{H}_{2} \mathrm{O}$ (bpy $=2,2^{\prime}$-bipyridine or bipyridyl; $\mathrm{Ln}=\mathrm{Nd}$, Ho, Er), were prepared according to a method described by Qian et al. [21]. The complexes were characterised by IR spectrometry and by $\mathrm{CHN}$ elemental analysis. The $\mathrm{SiO}_{2}$ sol-gel glasses incorporating lanthanide(III) bipyridyl complexes were obtained by hydrolysis of the monomeric precursor tetraethylorthosilicate (TEOS). For the acidcatalysed TEOS sol preparation, exactly measured volumes of TEOS (160 ml, obtained from Aldrich) and ethanol (80 $\mathrm{ml}$ ) were mixed. To this mixture was slowly added a concentrated aqueous $\mathrm{HCl}$ solution $(28 \mathrm{ml})$, resulting in a solution with $\mathrm{pH}=2$ and an $\mathrm{H}_{2} \mathrm{O}$ /precursor ratio of 2.2. This mixture was heated at reflux during 6 days and, after this period, divided into batches of $40 \mathrm{ml}$. [ $\left.\mathrm{Ln}(\mathrm{bpy})_{2}\right] \mathrm{Cl}_{3}$. $2 \mathrm{H}_{2} \mathrm{O}$ was dissolved in these solutions, so that the $\mathrm{Ln} / \mathrm{Si}$ ratios varied between 0.0028 and 0.015 . Of each solution, 4-ml quantities were divided over small glass vials (diameter $2.4 \mathrm{~cm}$, height $5 \mathrm{~cm}$ ) and the vials were closed by Parafilm. After 1 week, the Parafilm cover was perforated several times by a needle and the solvent was allowed to evaporate. After ca. 45 days, transparent monolithic glass samples were obtained, and most of them were free of cracks. In comparison to the wet gel, the glass sample shrinked, on average, over $90 \%$ in height and $35 \%$ in diameter. The glasses show the typical colours of the trivalent lanthanide ions [22]. 
The absorption spectra were measured on a Shimadzu UV3100 spectrophotometer. First, the samples were measured without heat treatment, secondly after heat treatment for $24 \mathrm{~h}$ at $100{ }^{\circ} \mathrm{C}$ in air and finally after an additional heat treatment of 4 days at $100{ }^{\circ} \mathrm{C}$ in air. The heat treatment was done by placing the glass samples in a furnace.

\section{Results and discussion}

Absorption spectra of the lanthanide-doped glass monoliths have been recorded between 380 and $1200 \mathrm{~nm}$. The spectra show narrow, weak absorption bands, typically for the $\mathrm{f}-\mathrm{f}$ transitions of trivalent lanthanide ions (Figs. 1-3). Each transition corresponds to a transition between two spin-orbit coupling levels. The majority of the transitions are induced electric dipole transitions, although some magnetic dipole transitions are observed. The dipole strengths of the transitions (expressed in debye ${ }^{2}, \mathrm{D}^{2}$ ) were extracted from the absorption spectrum, using the formula:

$D=\frac{1}{108.9 \times C \times d} \int \frac{A(\bar{\nu})}{\bar{\nu}} \mathrm{d} \bar{\nu}$,

where $C$ is the concentration of the lanthanide ion (mol $\left.1^{-1}\right), d$ is the optical path length $(\mathrm{cm}), A$ is the absorbance $\left[A=-\log \left(I / I_{0}\right)\right]$ and $\bar{\nu}$ is the wavenumber $\left(\mathrm{cm}^{-1}\right)$. The absorbance is related to the molar absorptivity, $\varepsilon$, via Lambert-Beer's law: $A=\varepsilon C d$. The dipole strength of a magnetic dipole transition can be calculated by using the 4f free-ion wave functions only. However, for the calculation of the dipole strengths of the induced electric dipole transitions, parameterisation is necessary. In the framework of the Judd-Ofelt theory $[19,20]$, the dipole strengths of induced electric dipole transitions can described by

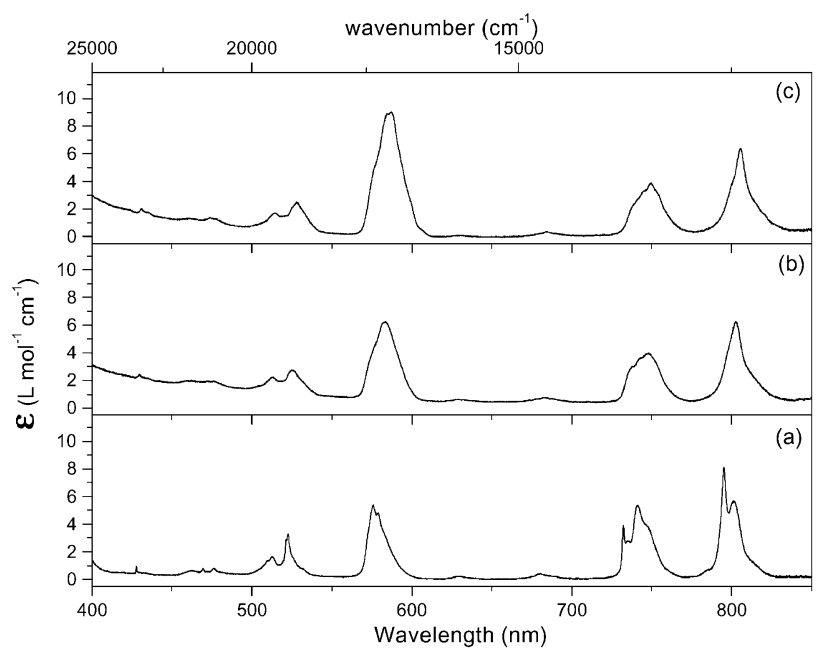

Fig. 1. Absorption spectrum of an $\mathrm{SiO}_{2}:\left[\mathrm{Nd}(\mathrm{bpy})_{2}\right] \mathrm{Cl}_{3}$ sol-gel glass at room temperature (a) before heat treatment, (b) after heat treatment for 24 $\mathrm{h}$ at $100{ }^{\circ} \mathrm{C}$, and (c) after heat treatment for 4 days at $100{ }^{\circ} \mathrm{C}$. The thickness of the sample was $0.27 \mathrm{~cm}$ and the $\mathrm{Nd}(\mathrm{III})$ concentration was $0.348 \mathrm{~mol} / 1$.

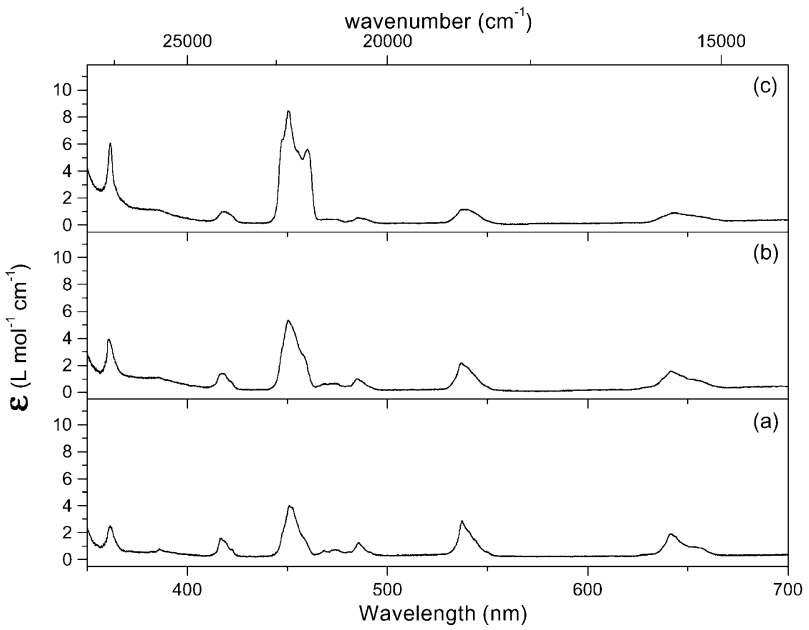

Fig. 2. Absorption spectrum of an $\mathrm{SiO}_{2}:\left[\mathrm{Ho}(\mathrm{bpy})_{2}\right] \mathrm{Cl}_{3}$ sol-gel glass at room temperature (a) before heat treatment, (b) after heat treatment for 24 $\mathrm{h}$ at $100{ }^{\circ} \mathrm{C}$, and (c) after heat treatment for 4 days at $100{ }^{\circ} \mathrm{C}$. The thickness of the sample was $0.22 \mathrm{~cm}$ and the Ho(III) concentration was $0.412 \mathrm{~mol} / 1$.

three phenomenological intensity parameters, $\Omega_{\lambda}(\lambda=2$, 4, 6):

$D=\frac{10^{36}}{2 J+1} \frac{\left(n^{2}+2\right)^{2}}{9 n} e^{2} \sum_{\lambda=2,4,6} \Omega_{\lambda}\left|\left\langle J\left\|U^{(\lambda)}\right\| J^{\prime}\right\rangle\right|^{2}$.

The factor $10^{36}$ is used for the conversion between $\mathrm{D}^{2}$ units and $\mathrm{esu}^{2} \mathrm{~cm}^{2}\left(1 \mathrm{D}=10^{-18} \mathrm{esu} \mathrm{cm}\right)$. The degeneracy of the ground state is equal to $2 J+1$. The factor $\left(n^{2}+\right.$ $2)^{2} /(9 n)$ takes into account that the lanthanide ion is not in a vacuum, but in a dielectric medium ( $n$ is the refractive index of the glass). Finally, the $\left|\left\langle J\left\|U^{(\lambda)}\right\| J^{\prime}\right\rangle\right|^{2}$ are the squared reduced matrix elements. The $\Omega_{\lambda}$ parameters are

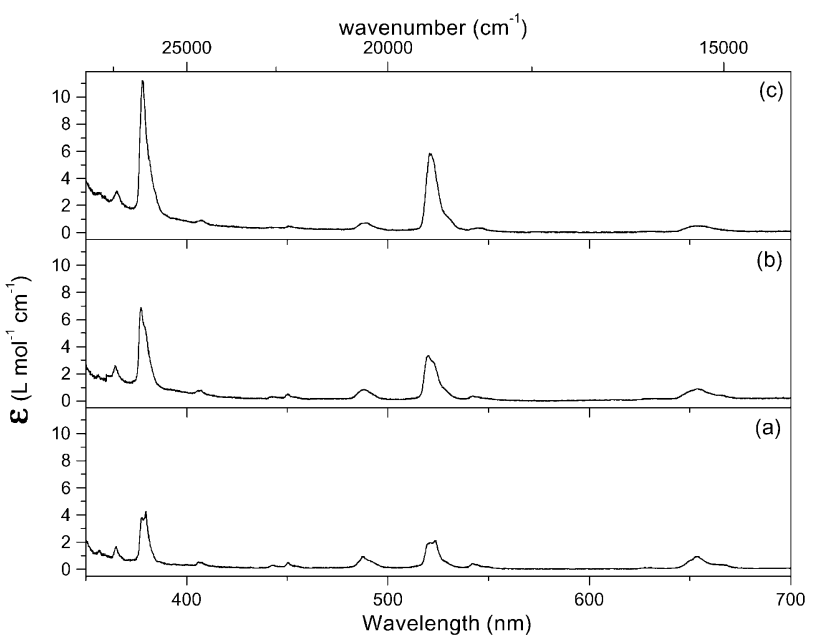

Fig. 3. Absorption spectrum of an $\mathrm{SiO}_{2}:\left[\mathrm{Er}(\mathrm{bpy})_{2}\right] \mathrm{Cl}_{3}$ sol-gel glass at room temperature (a) before heat treatment, (b) after heat treatment for 24 $\mathrm{h}$ at $100{ }^{\circ} \mathrm{C}$, and (c) after heat treatment for 4 days at $100{ }^{\circ} \mathrm{C}$. The thickness of the sample was $0.22 \mathrm{~cm}$ and the $\operatorname{Er}(\mathrm{III})$ concentration was $0.568 \mathrm{~mol} / 1$. 
Table 1

Judd-Ofelt parameters of the $\mathrm{SiO}_{2}:\left[\mathrm{Ln}(\mathrm{bpy})_{2}\right] \mathrm{Cl}_{3}$ sol-gel glasses before and after heat treatment

\begin{tabular}{|c|c|c|c|c|}
\hline Sample & Thermal history & $\Omega_{2}\left(10^{-20} \mathrm{~cm}^{2}\right)$ & $\Omega_{4}\left(10^{-20} \mathrm{~cm}^{2}\right)$ & $\Omega_{6}\left(10^{-20} \mathrm{~cm}^{2}\right)$ \\
\hline \multirow{3}{*}{ Nd glass } & No heat treatment & $0.5 \pm 0.2$ & $5.1 \pm 0.7$ & $5.1 \pm 0.3$ \\
\hline & $24 \mathrm{~h}$ at $100^{\circ} \mathrm{C}$ & $2.5 \pm 0.2$ & $4.4 \pm 0.3$ & $4.5 \pm 0.2$ \\
\hline & 4 days at $100^{\circ} \mathrm{C}$ & $3.9 \pm 0.3$ & $3.5 \pm 0.3$ & $4.2 \pm 0.2$ \\
\hline \multirow[t]{3}{*}{ Ho glass } & No heat treatment & $1.4 \pm 0.5$ & $1.5 \pm 0.7$ & $2.0 \pm 0.5$ \\
\hline & $24 \mathrm{~h}$ at $100^{\circ} \mathrm{C}$ & $2.9 \pm 0.5$ & $1.4 \pm 0.8$ & $1.8 \pm 0.5$ \\
\hline & 4 days at $100^{\circ} \mathrm{C}$ & $6.6 \pm 0.2$ & $1.2 \pm 0.4$ & $1.0 \pm 0.2$ \\
\hline \multirow[t]{3}{*}{ Er glass } & No heat treatment & $1.9 \pm 0.2$ & $0.7 \pm 0.3$ & $0.9 \pm 0.2$ \\
\hline & $24 \mathrm{~h}$ at $100^{\circ} \mathrm{C}$ & $3.5 \pm 0.3$ & $0.8 \pm 0.5$ & $1.0 \pm 0.3$ \\
\hline & 4 days at $100^{\circ} \mathrm{C}$ & $5.5 \pm 0.3$ & $0.8 \pm 0.5$ & $0.4 \pm 0.3$ \\
\hline
\end{tabular}

determined via a least squares fit, minimising the sum of the squares of the differences between experimental and calculated dipole strengths. The Judd-Ofelt parameters of the glass samples before and after heat treatment are summarised in Table 1.

The values of the Judd-Ofelt parameters for the glasses before heat treatment are quite low, comparable with the values of lanthanide ions in inorganic glasses or in aqueous solution. This is unexpected. Due to ligand polarisation, for lanthanide ions surrounded by organic ligands, a strong intensity enhancement is predicted (for lanthanide ions at a non-centrosymmetric site). Major changes were observed in the absorption spectra upon heat treatment. Most of the $\mathrm{f}-\mathrm{f}$ transitions showed roughly the same intensity (or a slight decrease), but the intensity of the hypersensitive transitions increased markedly. These hypersensitive transitions are the ${ }^{4} \mathrm{G}_{5 / 2} \leftarrow{ }^{4} \mathrm{I}_{9 / 2}$ transition of $\mathrm{Nd}$ (III) $(586 \mathrm{~nm})$, the ${ }^{5} \mathrm{G}_{6} \leftarrow{ }^{5} \mathrm{I}_{8}$ transition of $\mathrm{Ho}$ (III) (451 nm) and the ${ }^{2} \mathrm{H}_{11 / 2} \leftarrow{ }^{4} \mathrm{I}_{15 / 2}$ and ${ }^{4} \mathrm{G}_{11 / 2} \leftarrow{ }^{4} \mathrm{I}_{15 / 2}$ transitions of $\operatorname{Er}(\mathrm{III})$ (at 521 and $378 \mathrm{~nm}$, respectively). Hypersensitive transitions are known to be very sensitive to changes in the ligand environments (hence, their name). This behaviour is reflected in the values of the $\Omega_{\lambda}$ intensity parameters. The intensity of the hypersensitive transitions is mainly described by the $\Omega_{2}$ parameter, whereas the intensities of the other $\mathrm{f}-\mathrm{f}$ transitions are described by the $\Omega_{4}$ and $\Omega_{6}$ parameters. This means that upon heat treatment, the value of the $\Omega_{2}$ parameter increases strongly. The $\Omega_{4}$ parameter remains constant within the experimental error and the $\Omega_{6}$ parameter decreases slightly. The trend in the $\Omega_{6}$ parameter can be rationalised by the fact that the glass matrix is denser after removal of the water molecules and continuation of the polycondensation between the residual silanol groups, so that the matrix becomes more rigid. Based on a comparative study of Er(III)-containing complexes, Jørgensen and Reisfeld [23] concluded that the $\Omega_{6}$ parameter is related to the rigidity of the medium in which the lanthanide ion is embedded. Rigid matrices show low values for the $\Omega_{6}$ parameter. The rigidity of the matrix increases in the order coordination complexes $<$ halide vapours $<$ hydrated ions $<$ viscous solutions $<$ glasses $<$ crystalline mixed oxides. The fact that the lanthanide is in a glass matrix has a stronger effect on the $\Omega_{6}$ parameter than the fact that the lanthanide ions has organic ligands in its first coordination sphere. Therefore, the $\Omega_{6}$ parameter is more influenced by matrix effects than by effects on the closest environment of the lanthanide ion (this contrasts the behaviour of the $\Omega_{2}$ parameter). The $\Omega_{4}$ parameter is hardly influenced by heat treatment. Another feature of the heat treatment is that the absorption bands broaden and loose fine structure.

It should be mentioned that increase in luminescence intensity was observed by Qian et al. [21] after heat treatment at $100{ }^{\circ} \mathrm{C}$ of $\mathrm{Eu}(\mathrm{III})$ bipyridyl complexes in comparable sol-gel glasses. These authors contribute the intensity enhancement to in situ formation of the $\mathrm{Eu}(\mathrm{III})$ bipyridyl complex. Jin et al. [7] also find an increase in luminescence intensity of the same complexes, but now heating to $450{ }^{\circ} \mathrm{C}$.

\section{Conclusions}

Heat treatment has a marked influence on the absorption spectra of Ln(III) bipyridyl complexes incorporated in silica sol-gel glasses. Whereas the intensities of the majority of the $\mathrm{f}-\mathrm{f}$ transitions remain constant or decrease after heat treatment, the intensities of the hypersensitive transitions increase strongly. This is reflected by the Judd-Ofelt intensity parameters: $\Omega_{2}$ increases upon heat treatment and $\Omega_{4}$ remains more or less constant, whereas $\Omega_{6}$ decreases upon heat treatment. These observations can be explained by the fact that water is expelled out of the sol-gel matrix by heat treatment, followed by the proceeding of the polycondensation between the residual silanol groups. This results in a change in coordination sphere of the lanthanide ion and in a more rigid glass matrix. Lanthanide ions can be used as a spectroscopic probe to probe the structure of a sol-gel glass matrix. 


\section{Acknowledgements}

K.D. (research assistant) and K.B. (postdoctoral fellow) thank the F.W.O.-Flanders (Belgium) for financial support. Support by the K.U. Leuven (GOA 98/03) is acknowledged.

\section{References}

[1] D. Levy, L. Esquivias, Adv. Mater. 7 (1995) 120.

[2] C.J. Brinker, G.W. Scherrer, Sol-Gel Science, The Physics and Chemistry of Sol-Gel Processing, Academic Press, San Diego, 1990.

[3] R. Campostrini, G. Carturan, M. Ferrari, M. Montagna, O. Pila, J. Mater. Res. 7 (1992) 745.

[4] M. Ferrari, A. Piazza, M. Montagna, G. Carturan, R. Campostrini, J. Sol-Gel Sci. Technol. 2 (1994) 783.

[5] L.R. Mattews, X.J. Wang, E.T. Knobbe, J. Non-Cryst. Solids 178 (1994) 44

[6] G.D. Qian, M.Q. Wang, J. Phys. Chem. Solids 58 (1997) 375.

[7] T. Jin, S. Tsutsumi, Y. Deguchi, K. Machida, G. Adachi, J. Alloys Compd. 252 (1997) 59.

[8] T. Jin, S. Inoue, S. Tsutsumi, K. Machida, G. Adachi, J. Non-Cryst. Solids 223 (1998) 123.
[9] T. Jin, S. Inoue, K. Machida, G. Adachi, J. Alloys Compd. 265 (1998) 234.

[10] V. Bekiari, P. Lianos, Adv. Mater. 10 (1998) 1455.

[11] V. Bekiari, G. Pistolis, P. Lianos, Chem. Mater. 11 (1999) 3189.

[12] V. Bekiari, P. Lianos, P. Judenstein, Chem. Phys. Lett. 307 (1999) 310.

[13] W. Strek, J. Solkolnicki, J. Legendziewicz, K. Maruszewski, R. Reisfeld, T. Pavich, Opt. Mater. 13 (1999) 41.

[14] H.H. Li, S. Inoue, K. Machida, G. Adachi, Chem. Mater. 11 (1999) 3171.

[15] G.D. Qian, M.Q. Wang, J. Am. Ceram. Soc. 83 (2000) 703.

[16] C. Görller-Walrand, K. Binnemans, in: K.A. Gschneidner Jr., L. Eyring (Eds.), Handbook on the Physics and Chemistry of Rare Earths, vol. 25, North-Holland, Amsterdam, 1998, p. 101, Chap. 167.

[17] B. Viana, N. Koslova, P. Aschebourg, C. Sanchez, J. Mater. Chem. 5 (1995) 719

[18] J. Sokolnicki, B. Urbanski, J. Legendziewicz, J. Alloys Compd. 300-301 (2000) 450.

[19] B.R. Judd, Phys. Rev. 127 (1962) 750.

[20] G.S. Ofelt, J. Chem. Phys. 37 (1962) 511.

[21] G.D. Qian, M.Q. Wang, M. Wang, J. Photochem. Photobiol., A 107 (1997) 121

[22] K. Binnemans, C. Görller-Walrand, Chem. Phys. Lett. 235 (1995) 163.

[23] C.K. Jørgensen, R. Reisfeld, J. Less-Common Met. 93 (1983) 107. 